\title{
Study on the Dispersion of Lubricant Film from a Cylindrical Gearwheels with Helical Teeth by Vibrational Analysis
}

\author{
V. NIOLA*, S. SAVINO, G. QUAREMBA, C. COSENZA, M. SPIRTO, A. NICOLELLA \\ Department of Industrial Engineering \\ University of Naples Federico II \\ Via Claudio 21 - 80125, Naples, Italy
}

ITALY

\begin{abstract}
Gearwheels are a common mechanism for distributing torque from mechanical devices to other mechanical devices. The tribological state of gears reduces their performance and lifespan. This paper presents a signal-processing basis for evaluating the vibrational dynamics of a single pair of gears under various tribological situations. This method not only identifies the tribological state of the system, but also leads to different applications in several mechanical systems.
\end{abstract}

Key-Words: Gearwheels, Vibrational analysis, Lubrication

Received: April 25, 2021. Revised: October 18, 2021. Accepted: November 14, 2021. Published: December $21,2021$.

\section{Introduction}

In mechanical systems, assessing tribological state is a critical issue. Indeed, tribological state has a significant impact on system functionality, and a poor lubrication regime can result in system failure and damage. Gear lubrication is an engineering topic that has piqued researchers' curiosity for a long time. Proper lubrication provides for a smooth motion transmission from the driver to the driven gear, as well as a longer gear fatigue lifespan. As a result, scientists have used a variety of methods to investigate the dispersion of lubricants (as well as the lack of lubricants). H. Liu et al. investigated the effects of lubrication on gearing efficiency, demonstrating that oil and grease improve efficacy (at high temperatures as well), provide a longer lifespan (fatigue as well), and can reduce vibration and noise caused by gearing: among the several analyzed methods, jet lubrification produced the best results [1]. Z. Wang et al. proposed a new model for the analysis of transient mixed lubrication of bevel gears, emphasizing how this type of lubrication had a significant impact on stiffness and damping during contact between the teeth of the gearwheels, as these two parameters increased significantly as the transmission torque increased [2]. W. Cao et al. found that the temperature is higher at the contact point of the two spiral bevel gearwheels, claiming that this is due to a thinner lubricant film than the rest of the tooth, resulting in increased contact pressures and a reduction in the fatigue lifespan of the tooth [3]. By examining the key morphological features of the gearwheel teeth, $\mathrm{H}$. Wang et al. investigated contact wear for helical gears in the elastohydrodynamic mixed lubrication (EHL) regime: He confirmed that the most wear occurs at the tooth's height, which is increased by a high roughness; this impact can be significantly decreased with good lubrication [5]. H. Liu et al. compared the effects of high and low lubrication on the coefficient of friction in a cylindrical gear, demonstrating that with high lubrication, the average coefficient of friction initially increases and then gradually decreases, whereas with poor lubrication, the average coefficient of friction initially increases significantly and then flattens; furthermore, when the lubrication level is high, the film thickness grows as the rotation speed increases, whereas when the lubrication level is low, the film thickness increases just minimally [6]. V. Niola et al. used a non-linear analysis of vibrational signals to examine three different lubrication regimes of a gear, allowing them to recognize them and identify the presence of a damage on the gearwheel teeth [15]. 


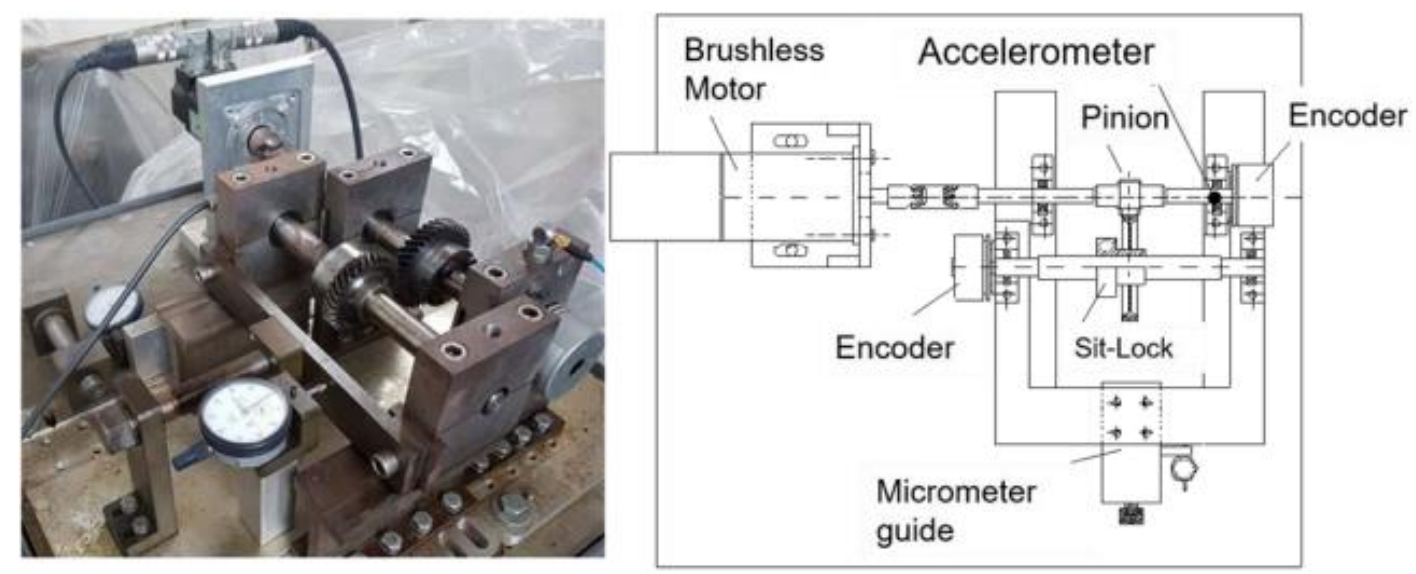

Fig. 1: Testbench

The goal of this project is to be able to evaluate the dispersion of the lubricant layer in a gear, for various speeds, using traditional approaches such as statistical indices analysis. These will be calculated for the vibrational signals obtained by an accelerometer. The methodology proposed has been already applied in the context of gear noise determination, cavitation phenomena and even for other mechanical systems [12-26].

\section{Experimental Approach}

A testbench consisting of the following components was set up: brushless electric motor, double cardan joint, two transmission shafts equipped with selfcentring clamping devices, four supports for bearings placed on the frame of the bench, two slides with micrometer screws and dial indicator (FVL 936), four radial ball bearings, an helical gear pair, two high resolution digital encoders (Baumer Electric BHF series), a support to place the electric motor. Knowing that the driving wheel has $33\left(z_{2}\right)$ teeth and the driven wheel has $37\left(z_{1}\right)$, the gear ratio $\varepsilon$ is 0.891 . The wheelbase was fixed at $65 \mathrm{~mm}$. The next figure shows the scheme of the test bench. Two types of testing were conducted:

- absence of lubrication

- poor lubrication: gearwheels were coated with a thin film of oil to achieve typical lubrication of the gear tooth contacts. For this test, SAE 75W90 oil was used.

Both groups of testing were performed at 500, 700, 900, and $1300 \mathrm{rpm}$. An accelerometer was linked to the system to capture the vibrations during wheel rotation, as seen in the next image. The signals were acquired for a total of 30 seconds, with 15 seconds of pause every 10 seconds $(10$ seconds of acquisition, 15 seconds of pause, 10 seconds of acquisition, 15 seconds of pause and 10 seconds of acquisition), in order to properly assess the lubricant dispersion during the rotation of the gearwheels. Knowing the system maximum frequency is 1600 $\mathrm{Hz}$, a sampling rate of $51200 \mathrm{~Hz}$ was determined in accordance with Nyquist's theorem.

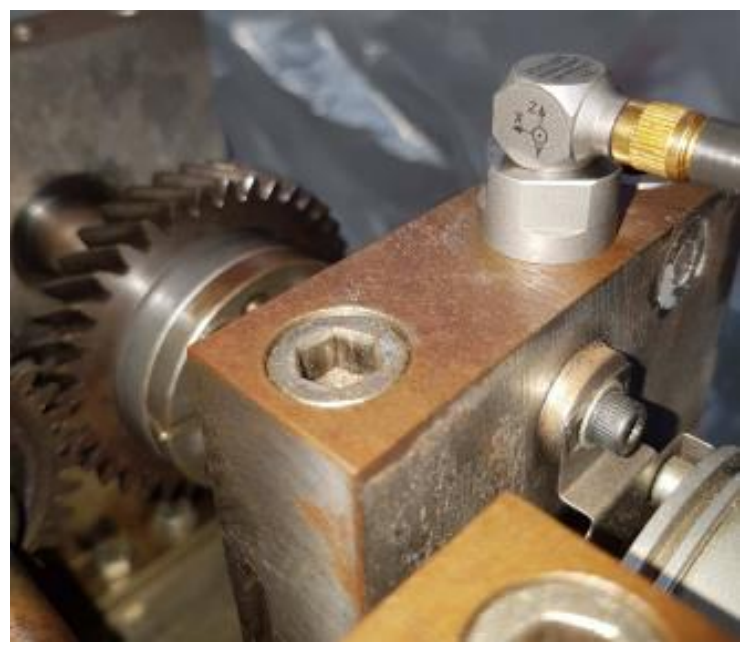

Fig. 2: Accelerometer position

\section{Results}

The results are only examined along the y-axis since they are the most significant. Below are the raw signals (Figure 3 and Figure 4). The amplitude of the signals increases (slightly) in case of lubrication, as can be seen by observing the eight signals and comparing the two different lubrication regimes with: this is exclusively due to the dispersion of the lubricant. There are no visible variations of this type in the case of absence of lubricant. In the following, the signals will be analyzed first by FFT and then by statistical indices. 

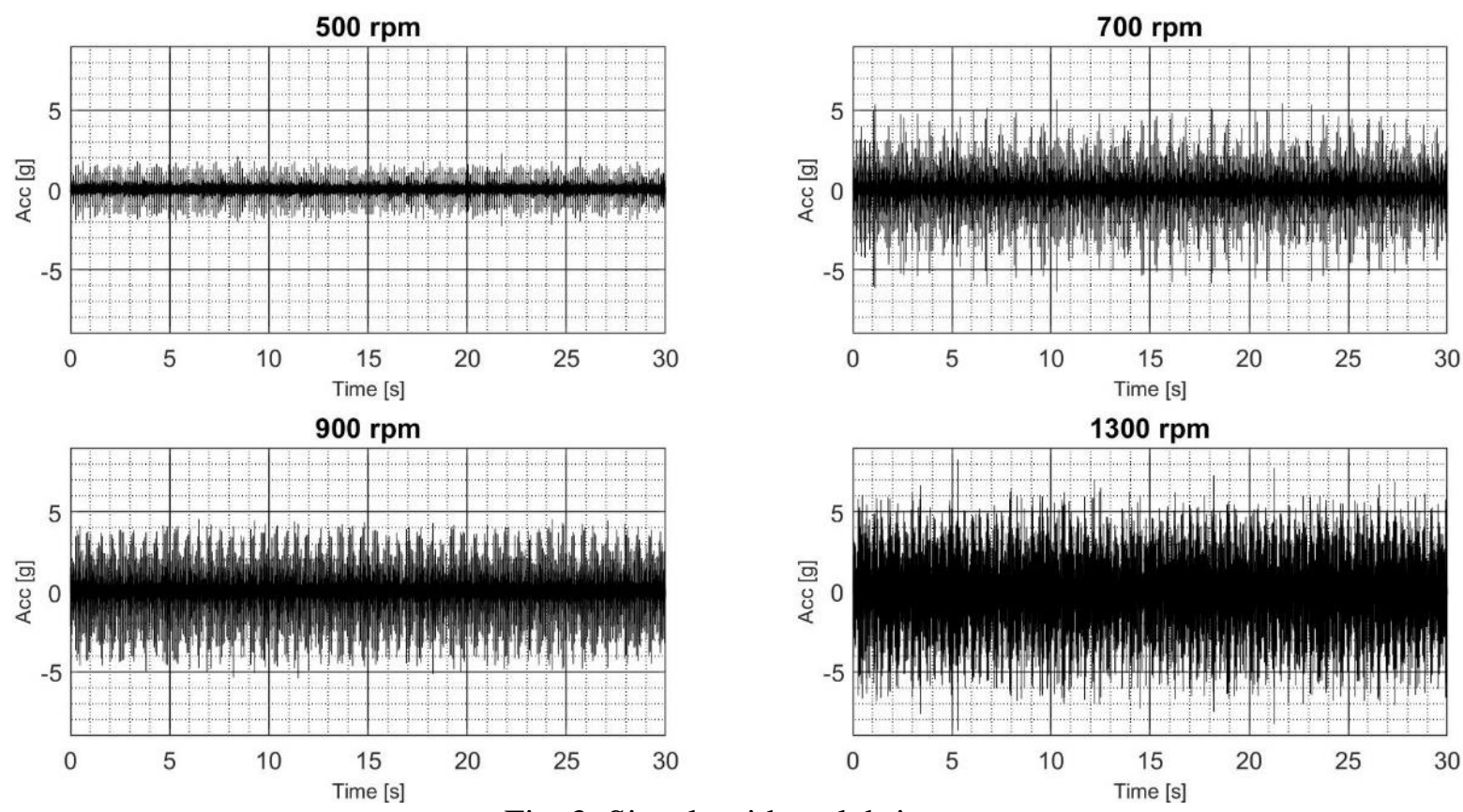

Fig. 3: Signals with no lubricant
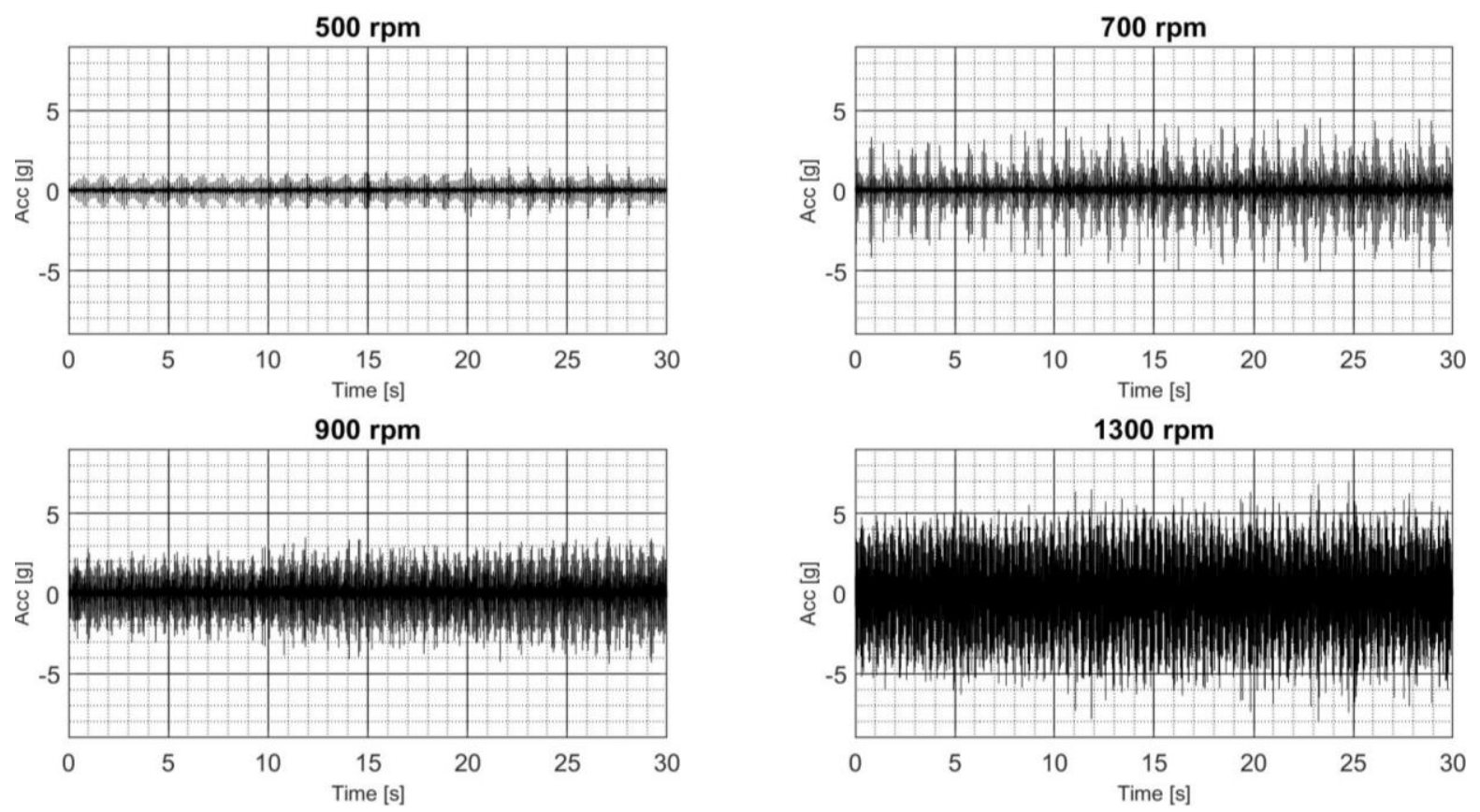

Fig. 4: Signals with lubricant

\subsection{Fast Fourier Transform}

The FFT of the raw signals is now performed. In the case of no-lubrication test, FFTs were calculated for the full signal (there is no lubricant, so there can be no dispersion); FFTs were calculated every 10 seconds of acquisition for each signal in the lubrification test (this choice is due to the 15 second pauses during acquisition). The different FFTs are displayed in Fig. 5 at Fig. 8. Observing the various diagrams, almost all energy, both with and without lubrication, is contained in the bandwidth between 0 and $5 \mathrm{kHz}$; this one grows up to around $10 \mathrm{kHz}$, when $1300 \mathrm{rpm}$ is reached. In the [0-5] kHz (and till to $10 \mathrm{kHz}$ for $1300 \mathrm{rpm}$ ), we found the rotation frequencies for the drive shaft $(8.33 \mathrm{~Hz}$ for $500 \mathrm{rpm}$, $11.67 \mathrm{~Hz}$ for $700 \mathrm{rpm}, 15 \mathrm{~Hz}$ for $900 \mathrm{rpm}, 21.67 \mathrm{~Hz}$ for $1300 \mathrm{rpm}$ ), the driven shaft and the brushless electric motor. Moreover, in such frequency range, we also found the noise. The bandwidth of harmonics increases with speed increasing. With the progressive increase in the rotation speed of the gears, we can also detect an increase in the amplitude of harmonics between 2.5 and $4.5 \mathrm{kHz}$. The FFTs of the raw signals were calculated for 
each second of acquisition to extract additional information from the spectra, and then the area subtended by these spectra was estimated. The obtained diagrams have the trend showed in Fig. 9. In Fig. 9 the curve representing the no oil regime has quiet constant behavior: this phenomenon is due to the lubrication absence. This behavior has a more chaotic trend, increasing the wheel angular rotation showing even an increase in the vibration state. This phenomenon is also connected to the surface defects and to the wear of the wheels. These aspects are more evident for high velocity regimes. When the two lubrication regimes are compared at the same rotational speed, the red curve (with lubricant) tends to the black one (without lubricant) in proportion to the lubricant dispersion. It is easy to observe how this tendency is slower at $500 \mathrm{rpm}$ and considerably faster, and almost totally reached, at $1300 \mathrm{rpm}$. The increase of this index indicates a rise of the bandwidth of the harmonics, as well as an amplitude growth and, therefore, an increasing energy content. Finally, the black curve, due to the absence of lubricant, is always higher than the red curve: the contact forces between the teeth are higher. As it can see, this index does not allow to visualize where the bandwidth is concentrated and, therefore, it is suggested use this index with the FFT previously seen.
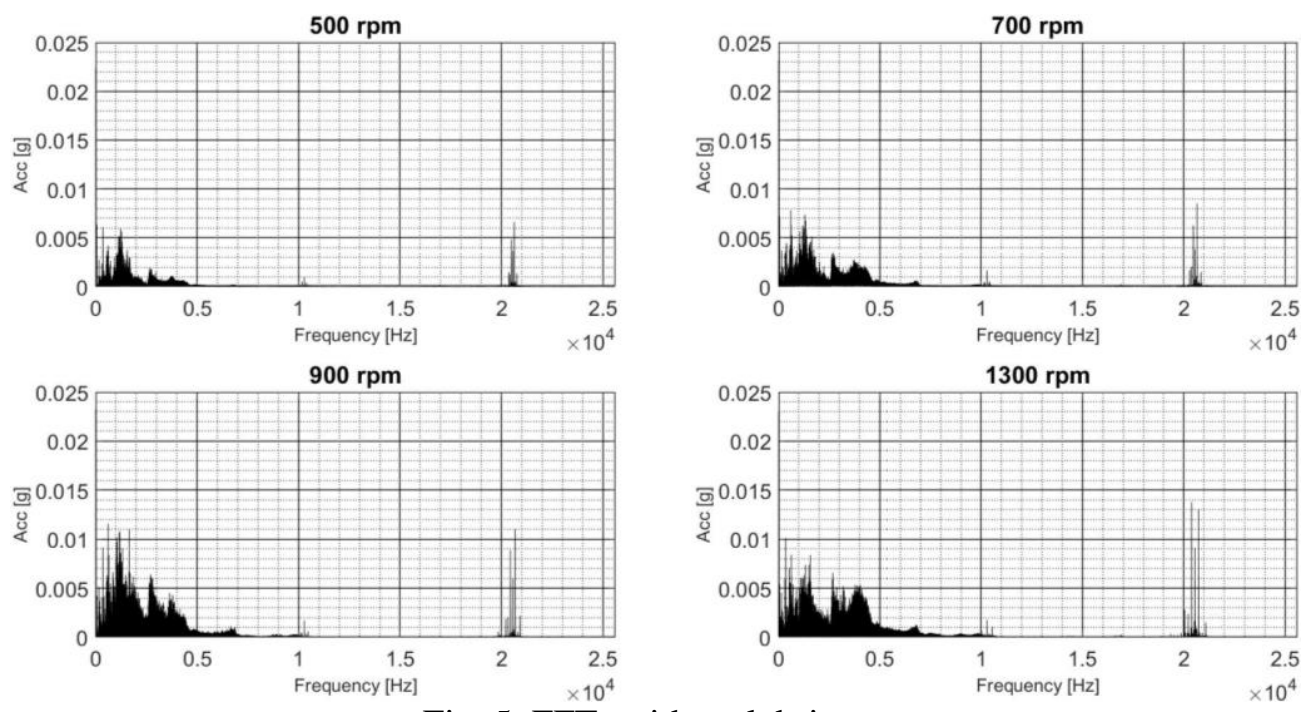

Fig. 5: FFTs with no lubricant
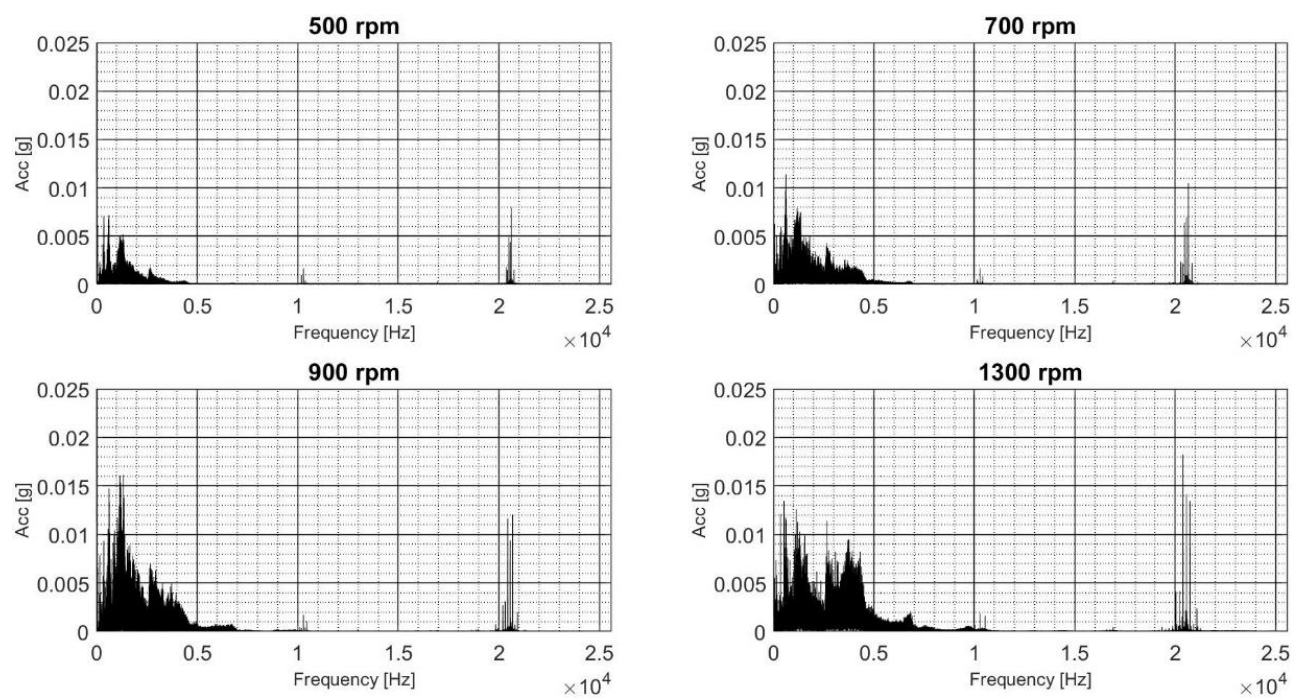

Fig. 6: FFTs with lubricant: first stint 

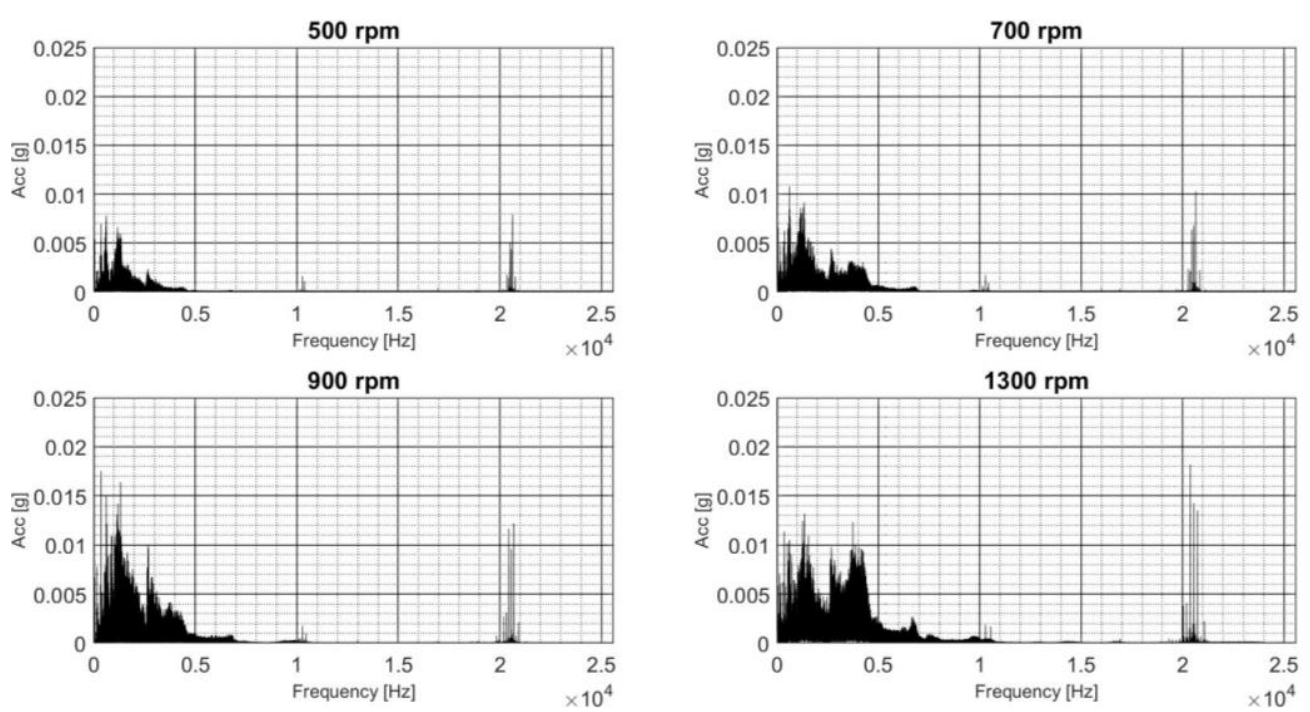

Fig. 7: FFTs with lubricant: second stint
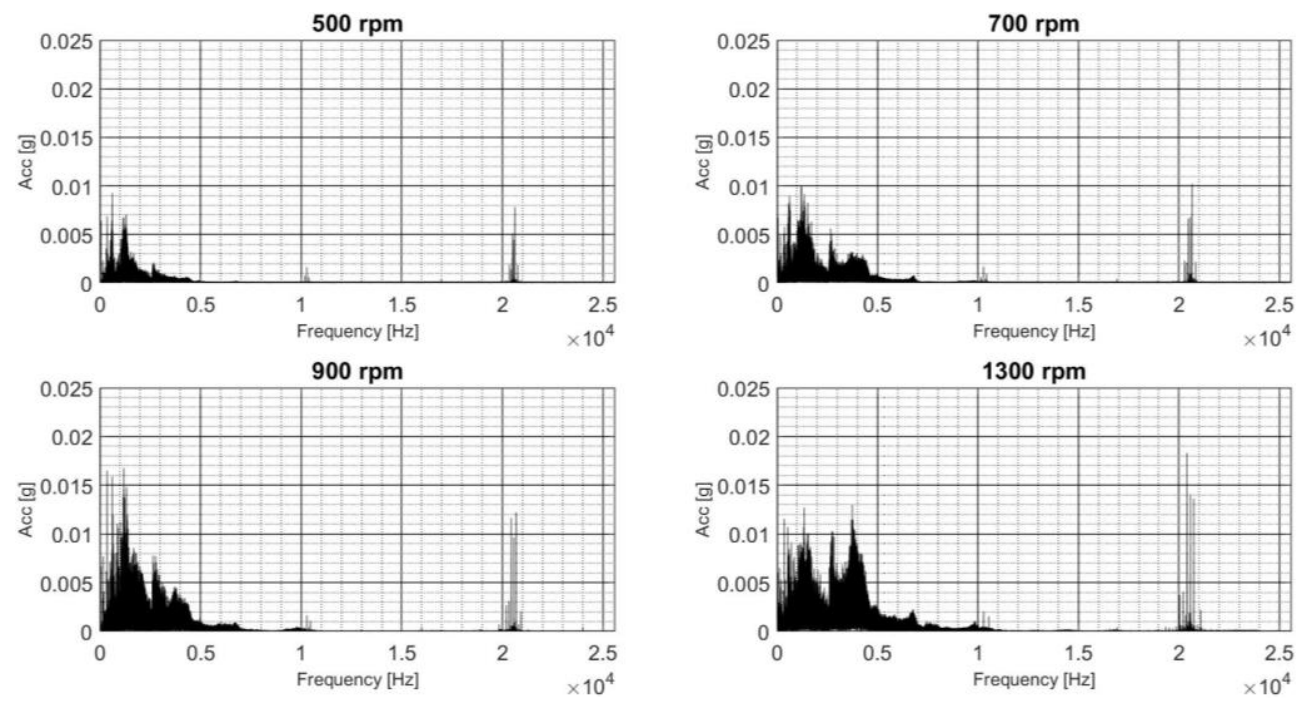

Fig. 8: FFTs with lubricant: third stint
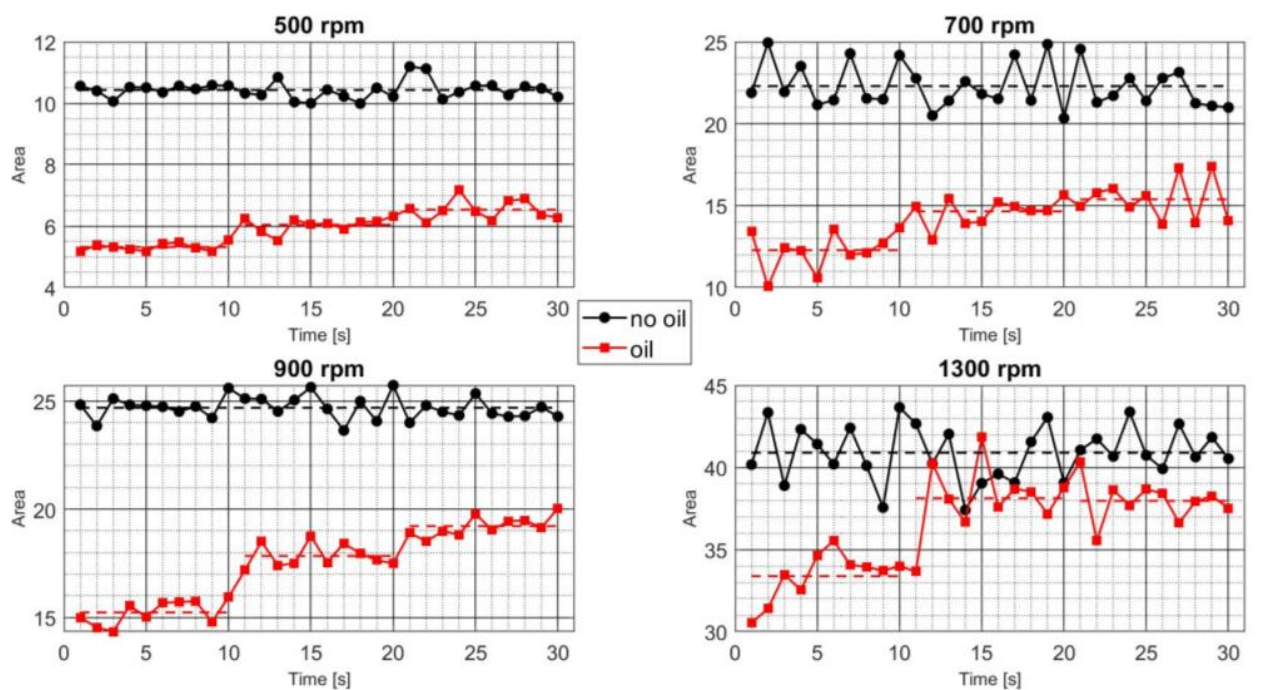

Fig. 9: Comparison between FFTs subtended areas

\subsection{Statistical Indices}

In the following, the raw signals will be analyzed using statistical indices. For each index computed on the generic signal, 30 values were obtained, i.e. 
one index value for each second of acquisition. The symbology used for the curves will always be the same: black curve and circular markers for the case

\subsubsection{Range}

The range $(\delta)[16]$ is defined as:

$$
\delta=x_{\max }-x_{\min }
$$

where $x_{\max }$ is the maximum value of signal $x$ and $x_{\min }$ the minimum value. Its trend is displayed in Fig. 10. The four graphs are in complete agreement with the observations made on the raw signals. In fact, it is possible to observe an increasing trend of the red curve proportional to the dispersion of the lubricant: this indicates that the amplitude of the signals is increasing. The red curve tends clearer to the black at higher speeds: this happens because at higher speeds lubricant dispersion is faster. In fact, in the case of $1300 \mathrm{rpm}$, the dispersion of the lubricant film is rapid, and already in the second period of acquisition (between 10 and 20 seconds), the average value of the red curve is very close to the black one. This increasing trend is much slower in the case of $500 \mathrm{rpm}$. Finally, it can be seen that the average values of the black curves increase as the speed of rotation increases, suitably with the increase of the number of impacts (between two teeth) in the generic second of acquisition.

\subsubsection{Root Mean Square Value}

The RMS [16] is defined as:

$$
R M S=\sqrt{\frac{1}{N} \sum_{i=1}^{N} x_{i}{ }^{2}}
$$

where $\mathrm{N}$ is the length of signal $x$ and $x_{i}$ is its generic component. Its trend is shown in Fig. 11. The trend of this index is very similar to the previous one (Fig. 10). In fact, as before, the red curve tends to the black one as the lubricant dispersion increasing. The progressive increase of the index indicates a progressive deterioration of the vibrational signal [10], i.e. that the forces acting on the system become higher. This is due to the decay of the lubricant film, which, as it disperses, is no longer able to significantly dampen the shocks between teeth. This is also confirmed by the increasing trend of the average values of the red curve, which also tends to increase like the relative curve. Finally, once again, it can be seen that the dispersion of the lubricant, so as the intensity of the shocks, is greater in the case of $1300 \mathrm{rpm}$ and less in the case of $500 \mathrm{rpm}$.

\subsubsection{Shape Factor}

The Shape Factor $(\mathrm{K})$ [16] is defined as: of with no lubricant, red curve and square markers for the case with lubrication, while the dashed lines stand for the average values.

$$
K=\frac{x_{m e d}}{\bar{x}}
$$

where $\bar{x}$ is the average value of signal $x$ and $x_{m e d}$ is the line such us to nullify integral:

$$
\int_{0}^{N} x d t
$$

where $\mathrm{N}$ is the length of signal $x$. In Fig. 12 is shown its trend. The graphs exhibit a fairly chaotic tendency, but it can also be observed that the curves are focused in a very small area, about between 0.50 and 0.57 . This index value range is typical of signals that evolve symmetrically in relation to their average value. It is interesting to underline how this symmetry is maintained despite the different lubrication regime, the dispersion of the lubricant film and the different rotation speeds considered.

\subsubsection{Logarithmic Entropy}

Logarithmic Entropy (LE) is defined as.:

$$
L E=\sum_{i=1}^{n} \log x_{i}{ }^{2}
$$

where $x_{i}$ is the generic component of the signal at the i-th instant; the logarithm is in natural base. In Fig. 13 it's possible to see the increasing trend of the red curve already seen for other indices (Range and RMS, Fig. 10 and 11) is once again repeated. The LE gives us a measure of the degree of order and disorder of the system: an increasing index indicates an increasing degree of disorder and, as in our case, an increase of dispersion of the signal values compared to their average one due to the dispersion of the lubricant film. The black curve, at the same speed of rotation, presents a fairly stable trend indicating how the degree of disorder is kept at about the same level, as well as presenting values always higher than the red curve: this is because there is no lubricant and, therefore, its dispersion over time. This "stability" tends to be lost at high speeds (see the case of $1300 \mathrm{rpm}$ ). These trends suggest that the last index is much more suitable than the others for evaluating the condition of the lubricant film on the gearwheels. Comparing this index with the Range and RMS, at the same speed of rotation, it can be seen that its growth is slower. 

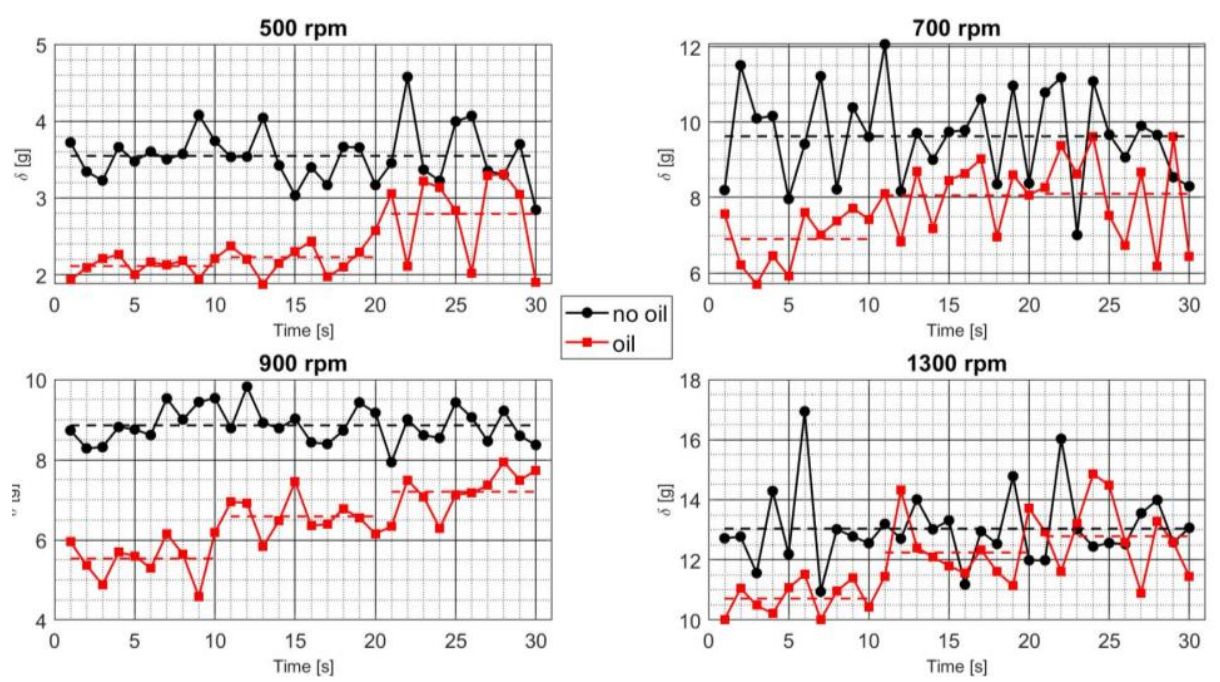

Fig. 10: Range trend
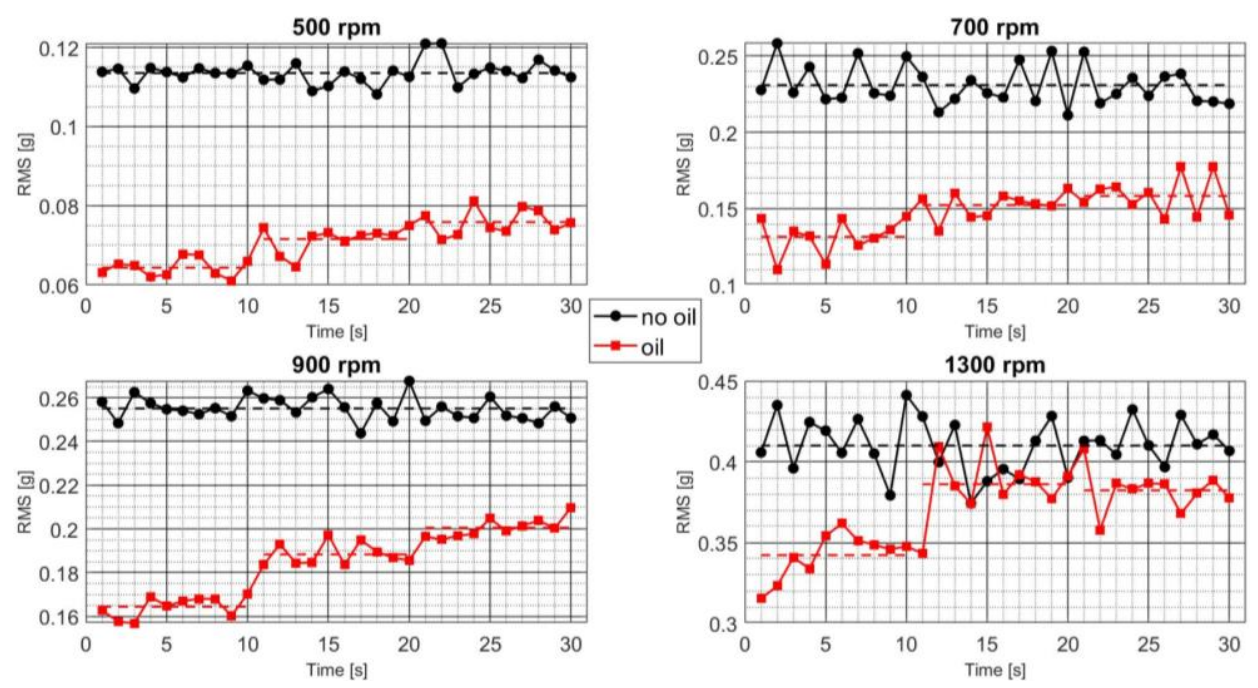

Fig. 11: RMS trend
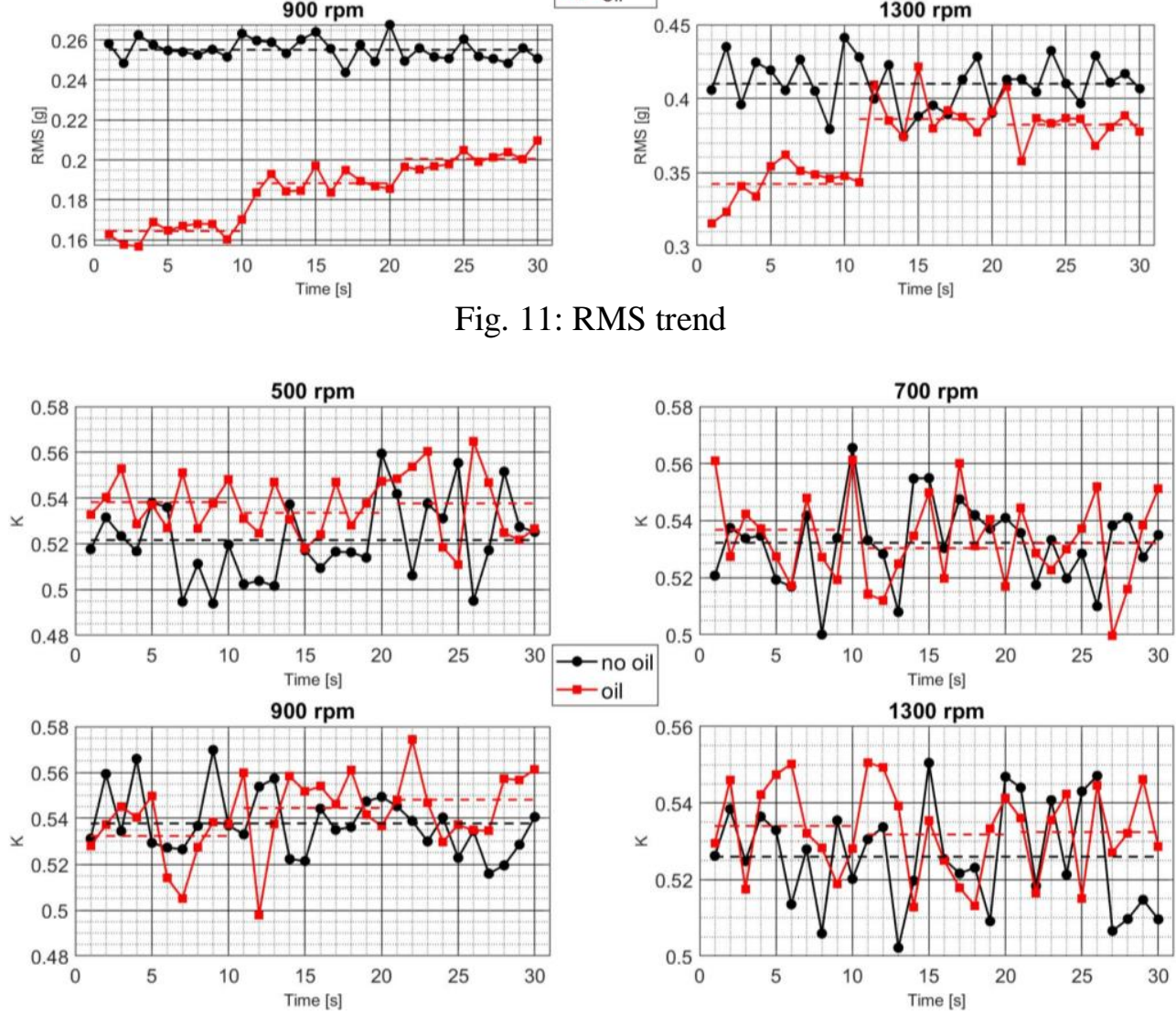

Fig. 12: Shape Factor trend 


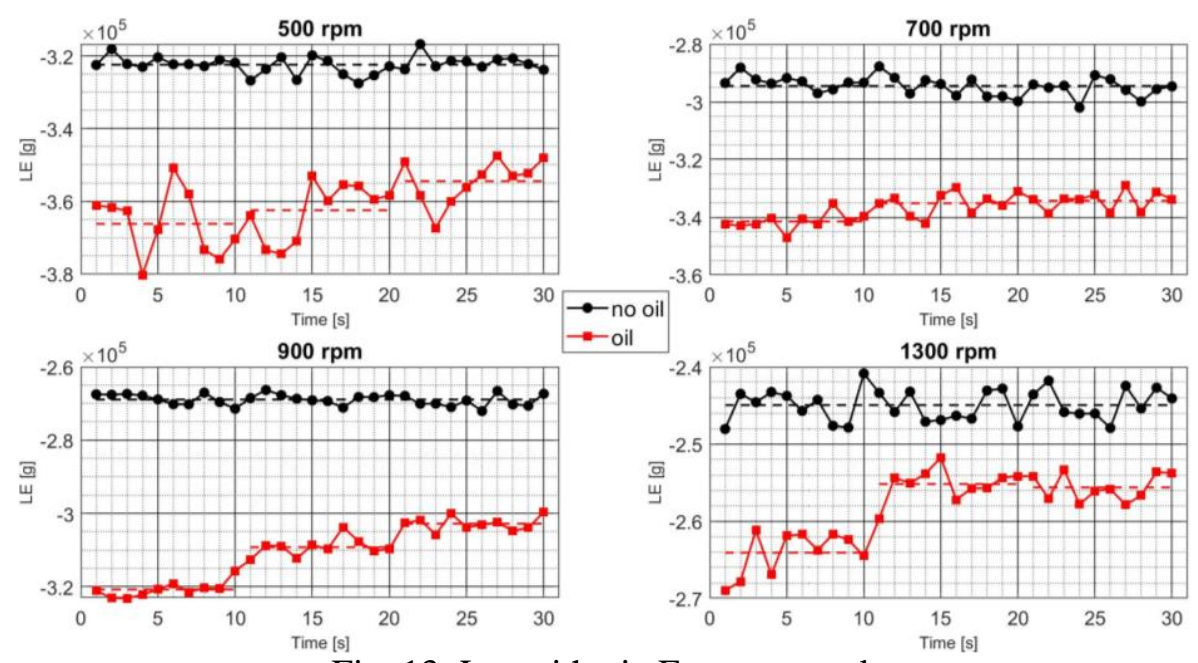

Fig. 13: Logarithmic Entropy trend.

\section{Conclusion}

In this paper, the study of lubricant film dispersion was addressed using two different methodologies. The study of the subtended areas by the FFTs provided clearer results in indicating the progressive dispersion of the lubricant film from the gear than the simple observation of the FFTs of the raw signals, although the simple index does not allow the visualization of the band of frequencies. The indices provide significant results regarding the progressive dispersion of the lubricant film, the increasing intensity of the forces due to impacts (see RMS index) and the amplitude grown of the vibrational signals (see Range index), all at the same speed of rotation. The study of the Shape Factor showed that the signal symmetrically develops itself than its average value, regardless of the lubrication level and the rotation speed of the system.

The Logarithmic Entropy (LE) assess the degree of order and disorder in the system: by this index, it is possible to see that the lubrification regime clearly tend to no-lubrication regime due to the progressive dispersion of the lubricant (in this case slower than the one seen for the Range and RMS). This method of analysis, in the future, could be implemented in real-time in order to evaluate the lubrication status of the system through the comparison between the vibrational signal, that is being acquired, and the basic one already acquired in conditions of absence of lubrication. Therefore, once the previously established threshold limit has been exceeded, it is possible to add a new lubrication amount if necessary.

\section{References:}

[1] H. Liu, H. Liu, C. Zhua, R.t G. Parker, Effects of lubrication on gear performance: A review, Mechanism and Machine Theory, Vol. 145,
2020,

DOI: 10.1016/j.mechmachtheory.2019.103701

[2] Z. Wang, W. Pu, X. Pei, W. Cao, Contact stiffness and damping of spiral bevel gears under transient mixed lubrication conditions, Springer, Vol. 123, pp. 359-71, 2018.

[3] W. Cao, W. Pu, J. Wanga, K. Xiao, Effect of contact path on the mixed lubrication performance, friction and contact fatigue in spiral bevel gears, Tribology International, Vol. 123, pp. 359-71, 2020.

[4] W. Cao, T. He, W. Pu, K. Xiao, Dynamics of lubricated spiral bevel gears under different contact paths, Springer, Vol. 10, pp. 47-67, 2022.

[5] H. Wanga, C. Zhoua,b , Y. Leia, Z. Liu, An adhesive wear model for helical gears in linecontact mixed elastohydrodynamic lubrication, Wear, Vol. 426-27, pp. 896-909, 2019.

[6] H. Liu, C. Zhu, Z. Sun, C. Song, Starved lubrication of a spur gear pair, Tribology International, Vol. 94, pp. 52-60, 2016.

[7] M. Hammami, R. Martins, M. S. Abbes, M. Haddar, J. Seabra, Axle gear oils: Friction behaviour under mixed and boundary lubrication regimes, Tribology International, Vol. 116, pp. 47-57, 2017.

[8] Z. Xiaoa, C. Zhoua, Z. Li , M Zhenga, Thermomechanical characteristics of high-speed and heavy-load modified gears with elastohydrodynamic contacts, Tribology International, Vol. 131, pp. 406-14, 2019.

[9] A. Ziegltrum, T. Lohner, K. Stahl, TEHL simulation on the influence of lubricants on load-dependent gear losses, Tribology International, Vol. 113, pp. 252-61, 2017. 
[10] V. Niola, G. Quaremba, Sistemi Vibrazionali Complessi, Teoria, Applicazioni e Metodologie Innovative di Analisi, Nane Edizioni, 2015.

[11] V. Niola, G. Quaremba, C. Cosenza, L. Russo, S. Savino, Comparison of linear methodologies and not for the evaluation of the vibrational morphodynamics of a pair of gears in different tribological conditions, Journal of Physics: Conference Series. Vol. 1589. No. 1, 2020, DOI 10.1088/1742-6596/1589/1/012010.

[12] Niola, V.; Rossi, C.; Savino, S.; Troncone, S. An Underactuated Mechanical Hand: A First Prototype. In Proceedings of the 2014 23rd International Conference on Robotics in AlpeAdria-Danube Region (RAAD), Smolenice, Slovakia, 3-5 September 2014; pp. 1-6.

[13] Cosenza, C.; Niola, V.; Savino, S. Modelling friction phenomena in an underactuated tendon driven finger by means of vision system device data. In Mechanisms and Machine Science; 2019; Vol. 68, pp. 297-306.

[14] Brancati, R.; Cosenza, C.; Niola, V.; Savino, S. Experimental measurement of underactuated robotic finger configurations via 442 RGB-D sensor. In Mechanisms and Machine Science, Vol. 67, pp. 531-537, 2019, DOI 10.1007/978-3-030-00232-9_56

[15] Cosenza, C.; Niola, V.; Savino, S. Underactuated finger behavior correlation between vision system based experimental tests and multibody simulations. In Mechanisms and Machine Science; Springer: Cham, Switzerland, 2019; Volume 66, pp. 49-56

[16] Niola V, Spirto M, Savino S, Cosenza C, Vibrational analysis to detect cavitation phenomena in a directional spool valve, JoMac, Vol. 22, No. 01, pp. 11-16, 2021, ISSN 15908844.

[17] Brancati R Capasso R, Niola V, Quaremba G, Rocca E, and Savino S, Nonlinear methodology for assessing vibrational dynamics of a single gear pair under different tribological conditions, Mechanisms and Machine Science, Vol. 73, pp 4127-34, 2019.

[18] Niola V., Quaremba G., Avagliano V., Vibration monitoring of gear transmission, 9th WSEAS International Conference on Simulation, Modelling and optimization, pp 7479, 20 , ISSN: 1790-2769.
[19] Niola V., Avagliano V., Quaremba G., The Problem of GWN, Mathematical Methods and Techniques in Engineering and Environmental Science, Vol. , No. , pp 363-70, 20 , ISBN: 9781-61804-046-6.

[20] Niola V., Avagliano V., Quaremba G., The Gear Whine Noise, Recent Researches in Geography, Geology, Energy, Environment and Biomedicine, Vol. , No. , pp 445-50, 20 , ISBN: 978-1-61804-022-0.

[21] Niola V and Quaremba G 2011 The Gear Whine Noise: the influence of manufacturing process on vibro-acoustic emission of gear-box Proceeding of 10th WSEAS international conference on electronics, hardware, wireless and optical communications, and 10th WSEAS international conference (Cambridge, UK: World Scientific and Engineering Academy and Society (WSEAS)) pp 175-179, 2011,

[22] Niola V., Quaremba G., Aniello F., Gear noise detection. An integrated method using Fourier and Wavelet approach, 6th WSEAS International Conference on SYSTEM SCIENCE and SIMULATION in ENGINEERING, Venice, Italy, November, pp. 21-23, 2007.

[23] Mengying Ding, Yali Dong, "Stability Analysis and Robust $\mathrm{H} \infty$ Filtering for Discretetime Nonlinear Systems with Time-varying Delays," WSEAS Transactions on Systems, Vol. 20, pp. 281-288, 2021

[24] Seda Postalcioglu, "Wavelet Transform Based Feature Extraction for EEG Signal Classification," WSEAS Transactions on Computers, Vol. 20, pp. 199-206, 2021

[25] Boris Shumilov, "Shifted Cubic Spline Wavelets with Two Vanishing Moments on the Interval and a Splitting Algorithm," WSEAS Transactions on Systems, Vol. 19, pp. 149-158, 2020.

[26] Suboh Alkhushayni, Taeyoung Choi, Du'a Alzaleq, "Data Analysis Using Representation Theory and Clustering Algorithms," WSEAS Transactions on Computers, Vol. 19, pp. 310320, 2020.

Creative Commons Attribution License 4.0 (Attribution 4.0 International, CC BY 4.0)

This article is published under the terms of the Creative Commons Attribution License 4.0 https://creativecommons.org/licenses/by/4.0/deed.en US 